\title{
THYROID GLAND
}

\section{Vandetanib for thyroid cancer}

Vandetanib, a tyrosine kinase inhibitor of RET, VEGFR and EGFR signalling, is effective in patients with locally advanced or metastatic differentiated thyroid cancer and could improve progression-free survival (PFS) in these patients, report Martin Schlumberger and colleagues in Lancet Oncology. Previous studies showed that vandetanib treatment is effective in patients with advanced medullary thyroid cancer, which led to the drug's approval by the FDA and European Medicines Agency for use in this population. However, no results of a randomised placebo-controlled study of vandetanib in patients with differentiated thyroid cancer had been previously reported.

Schlumberger and colleagues randomly assigned 145 patients (aged $\geq 18$ years) with histologically confirmed locally advanced or metastatic differentiated thyroid cancer to receive either $300 \mathrm{mg}$ of vandetanib per day $(n=72)$ or placebo $(n=73)$ at 16 European medical centres between September 2007 and October 2008. "At the time, there was no other trial in which these patients were enrolled, and to provide original data we decided not to do a single-arm phase II study, but a randomised placebo-controlled study," says Schlumberger.

The patients selected were individuals who were not suitable to receive radioiodine treatment, as they had experienced disease progression following previous radioiodine therapy or had one or more lesions without detectable radioiodine uptake. Patients who were receiving chemotherapy or radiotherapy within 4 weeks before the initiation of the study and those who had been previously treated with vandetanib were excluded.

The primary end point was PFS, defined as the time from randomisation to the date of objective disease progression or death from any cause. Patients were followed up until $\geq 50 \%$ had died or until all patients stopped treatment owing to disease progression, stable disease for 12 months, or adverse events. RECIST assessments were carried out at baseline and every 12 weeks thereafter, and serum TSH and thyroglobulin levels were measured at 4 weeks, 8 weeks, and then every 12 weeks until disease progression was observed.

After a median follow-up of 18.9 months in the vandetanib group and 19.5 months in the placebo group, disease progression was detected in $78 \%$ of patients in the former group versus $84 \%$ in the latter. Although overall survival was not different between the two groups, median PFS was significantly shorter in the placebo group (5.9 months) than in the vandetanib group (11.1 months). Histological analyses showed that improvement in PFS was greater in patients with papillary thyroid cancer than in those with other cancer subtypes, but this difference was not significant. RECIST assessments revealed that vandetanib treatment reduced response time from 11.4 months to 9.0 months. Moreover, disease was controlled in $57 \%$ of patients in the vandetanib group versus $42 \%$ in the placebo group. Nevertheless, early tumour responses (measured by ${ }^{18} \mathrm{~F}$-fluorodeoxyglucose PET scans) and biochemical responses (measured by serum analyses) were not different between the two groups.

Overall, vandetanib therapy was well tolerated. The most common adverse events included diarrhoea, hypertension and corrected QT interval prolongation; however, no serious cardiac complications were noted.

"This is the first randomized study to show an improvement in PFS with vandetanib in this setting and these data confirm that tyrosine kinase inhibitors are effective in terms of objective response rate and PFS," states Schlumberger. "Although the response rate with vandetanib is lower than that reported with other kinase inhibitor drugs, the improvement in PFS and disease control suggest that vandetanib is an effective treatment for advanced differentiated thyroid carcinoma," he explains. Schlumberger and colleagues are now trying to identify the subgroup of patients for whom vandetanib is the most effective.

Iley Ozerlat

Original article Leboulleux, S. et al. Vandetanib in locally advanced or metastatic differentiated thyroid cancer: a randomised, double-blind, phase 2 trial. Lancet Oncol. doi:10.1016/S14702045(12)70335-2 